$\underline{\text { Research Article }}$

\title{
Prospective 18 month surveillance study of MRSA colonization in an Orthopedic unit in Sri Lanka.
}

\author{
V Thevanesam ${ }^{1}$, H J Suraweera ${ }^{2}$, P Kannangara ${ }^{1}$, I K B Weerasekera ${ }^{1}$, H M W Abeywardena ${ }^{1}$, \\ E W M A Ekanayake ${ }^{1}$, T M Gamage ${ }^{1}$, LVC Liyanapathirana ${ }^{1}$ \\ Sri Lankan Journal of Infectious Diseases 2013 Vol.3(1);9-14 \\ DOI: http://dx.doi.org/10.4038/sljid.v3i1.4046
}

Key words: MRSA, orthopaedic, surveillance

\begin{abstract}
The prevalence and acquisition of MRSA colonization in an Orthopaedic Unit in Sri Lanka were studied over an 18 month period. 260 of 1684 patients screened on admission were colonized with MRSA. Patients who were negative at preliminary screening were rescreened at weekly intervals during their hospital stay. Of 1424 patients who were rescreened, 170 acquired MRSA. This information is beneficial in planning and implementing suitable infection control procedures in Sri Lankan hospitals.
\end{abstract}

\section{Introduction}

The increasing prevalence of Methicillin Resistant Staphylococcus aureus (MRSA) infections and the difficulty in containing the spread of the organism is a concern to many. The menace of MRSA has increased to such an extent now that community acquired MRSA infections are increasing in prevalence in addition to the ever increasing rates of hospital acquired MRSA infections. ${ }^{1,2}$ Colonization with MRSA is recognized as a risk factor that precedes infections with MRSA. ${ }^{3,4}$ In addition, colonized individuals act as reservoirs within health care facilities in the spread of the organism. ${ }^{1}$

Longitudinal studies to determine the rates of acquisition of MRSA are important to determine the risk factors associated with colonization as well as to determine the value of interventions such as isolation, cohorting of patients, appropriate prophylactic antibiotic use and hand washing after single patient contact.

${ }^{1}$ Department of Microbiology, Faculty of Medicine, University of Peradeniya, Sri Lanka.

${ }^{2}$ Orthopaedic Unit, Teaching Hospital, Peradeniya

Address for correspondence: Prof V Thevanesam. Department of Microbiology, Faculty of Medicine, Peradeniya, Sri Lanka Email: vasanthithevanesam@yahoo.com Tele No: +94773157451 
MRSA associated infections in orthopedic patients, particularly in those who undergo joint replacement surgery, is associated with significant morbidity and health care associated expenses. Many hospitals therefore recommend screening, either universally or based on risk factors followed by decolonization, taking into consideration the associated financial implications. 5

This study was carried out to determine the prevalence of MRSA colonization on admission and the rate of acquisition of MRSA during the stay in an orthopaedic unit of a teaching hospital in the Central Province of Sri Lanka.

\section{Methods}

\section{Patients and ward setting}

Patients admitted to the Orthopaedic Unit, Teaching Hospital, Peradeniya, Sri Lanka from May 2003 to November 2004 were considered for inclusion in the study. The only exclusion criterion was discharge or transfer from the unit within 24 hours of admission. The orthopaedic unit is a 62 bedded ward comprising of a central lobby, a male ward with 36 beds and a female ward with 26 beds. The outpatient clinic is held in a large 5 bedded room at the entry to the female ward.

\section{Screening methods.}

Patients were screened between 24 and 36 hours of admission. Swabs for screening were taken from nostrils, axillae, throat and perineum in male patients and all sites except perineum in female patients. Bedside inoculation into salt broth was followed after 24 hours incubation at $35^{\circ} \mathrm{C}$ by subculture onto Mannitol Salt Agar. Yellow colonies were identified using standard methods as $S$ aureus and methicillin sensitivity tested using the NCCLS method. Acquisition of MRSA during the patient's stay was determined by re-screening MRSA negative patients at weekly intervals until their discharge from the unit.

\section{Results}

Three thousand one hundred and fifteen (3115) patients were admitted to the unit during the study period. Of this number, 1431 (45.9\%) patients were discharged or transferred to another ward within 24 hours of admission and therefore excluded from the study. MRSA screening was carried out on the remaining 1684 patients.

A total of 260 patients out of the 1684 screened (15.4\%) were MRSA positive on admission. Of 1420 patients who were MRSA negative on admission, 886 remained in the ward for periods longer than a week, allowing re-screening at weekly intervals. 170 of these patients acquired MRSA during their stay in the ward.

The rate of MRSA acquisition by patients admitted to the ward is expressed in 2 ways. The number of in-ward patients who acquired MRSA each month is expressed by dividing the 
number of patients who acquired MRSA during the given month by the number of patients without MRSA on admission during that month (Table 01).

Table 1 - Monthly summary of data (data was not collected in September 2004)

\begin{tabular}{|c|c|c|c|c|c|c|}
\hline Manth & $\begin{array}{c}\text { Total number } \\
\text { of } \\
\text { patients } \\
\text { screened }\end{array}$ & $\begin{array}{c}\text { MRSA } \\
\text { colonized an } \\
\text { admission } \\
\text { (п) }\end{array}$ & $\begin{array}{l}\text { \% colanized } \\
\text { on admissian } \\
(\%)\end{array}$ & $\begin{array}{c}\text { MRSA negative } \\
\text { on admission }\end{array}$ & $\begin{array}{l}\text { MRSA positive } \\
\text { on } \\
\text { rescreening } \\
\text { (п) }\end{array}$ & $\begin{array}{c}\text { Monthly \% of } \\
\text { MRSA } \\
\text { acquisition }\end{array}$ \\
\hline I(May 2003) & 150 & 52 & 34.7 & 98 & 15 & 15.3 \\
\hline 2(June 2003) & 78 & 24 & 30.8 & 54 & 9 & 伯.7 \\
\hline 3(July 2003) & 100 & 26 & 26.0 & 74 & 10 & 13.5 \\
\hline 4(August 2003) & 97 & 16 & 16.5 & 81 & 0 & 0.0 \\
\hline 5 (September 2003) & 102 & 23 & 22.5 & 89 & 8 & 9.0 \\
\hline G (Dctober 2003) & 别 & 10 & 11.6 & 76 & 19 & 25.0 \\
\hline 7 (November 2003) & 89 & 5 & 5.6 & 84 & 10 & 11.9 \\
\hline 8 (December 2003) & 112 & 21 & 18.8 & 91 & 10 & 11.0 \\
\hline ५ (January 2004) & 88 & 10 & 11.4 & 78 & 6 & 7.6 \\
\hline 10 (Febгuaгу 2004) & 75 & 9 & 12.0 & 66 & 3 & 4.5 \\
\hline II (March 2004) & 97 & 13 & 13.4 & 84 & 15 & 17.9 \\
\hline I2 (April 2004) & 吅 & 15 & 18.8 & 65 & 8 & 12.3 \\
\hline 13 (May 2004) & 77 & 8 & 10.4 & 69 & G & 13.0 \\
\hline 14 (June 2004) & 104 & 12 & 11.5 & 92 & 17 & 18.5 \\
\hline 15 (July 2004) & 72 & 5 & 6.9 & 67 & 8 & 11.9 \\
\hline 16 (August 2004) & 75 & 4 & 5.3 & 71 & 8 & 11.3 \\
\hline 17 (पctober 2004) & 114 & 2 & 1.8 & 112 & 8 & 7.1 \\
\hline 18(November 2004) & 88 & 5 & 5.7 & 83 & 7 & 8.4 \\
\hline Total & 1684 & 260 & 15.4 & 1424 & 170 & 11.9 \\
\hline
\end{tabular}

The likelihood of individual patients acquiring MRSA during their stay in the ward is expressed as the percentage of patients who became positive for MRSA at consecutive weekly retesting which also reflects the relationship between the length of hospitalization and MRSA acquisition (Table 02).

Table 2 - Acquisition rates with weekly screening

\begin{tabular}{cccc}
\hline Week of retesting & Number of patients retested & Number of patients becoming MRSA positive & Acquisition rate (\%) \\
\hline 1 & 513 & 80 & 17.2 \\
2 & 217 & 43 & 19.8 \\
3 & 86 & 21 & 24.4 \\
4 & 36 & 11 & 30.6 \\
5 & 23 & 5 & 21.7 \\
6 & 6 & 1 & 16.6 \\
7 & 4 & 1 & 25 \\
8 & 1 & 0 & 0 \\
\hline
\end{tabular}


Of the 886 rescreened patients, the majority (82.3\%) remained in the ward for 2 weeks or less. The risk of acquiring MRSA during the in-ward stay ranged from 16.6\% to $30.6 \%$ as shown in Table 2. A Kaplan Meier survival curve was constructed with MRSA colonization as the outcome. As seen in the graph (Figure 1), $50 \%$ of patients who were not colonized with MRSA at admission and remained as inpatients were colonized by week 3 . The graph also clearly shows that the longer the duration of hospital stay, the higher the chance of acquiring MRSA.

\section{Figure 1: Acquisition of MRSA related to length of hospital stay}

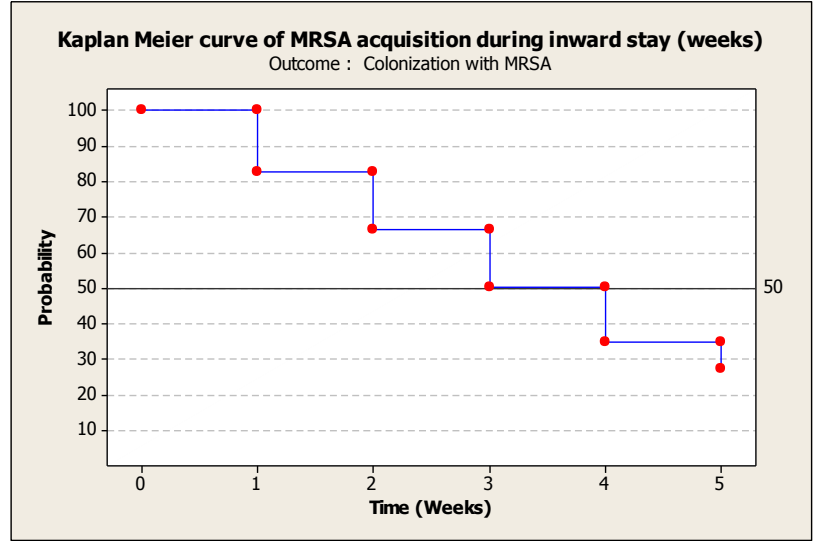

\section{Discussion}

Our study shows that a considerable proportion of patients $(15.4 \%)$ admitted to a tertiary care orthopaedic centre in a relatively resource poor country were colonized with MRSA on admission. This is higher than reported from another centre with similar background from Sri Lanka. ${ }^{6}$ This may be due to differences in the screening procedure, patient population or community prevalence of MRSA. Worldwide rates of MRSA colonization reported vary according to the methodologies used by researchers., ${ }^{7,8,9}$

Recommendations for MRSA screening varies from universal screening of all patients admitted to orthopaedic units to screening dependent on the presence of risk factors associated with MRSA colonization. Availability of resources would be a major factor in deciding screening policy in resource strapped health care facilities. Many studies have been carried out to determine whether colonization with MRSA is associated with an increased risk of infection with contradictory outcomes. Some studies have shown significant differences in infection rates in patients colonized with MRSA when compared to those without $S$ aureus colonization or those colonized with methicillin sensitive $S$ aureus. ${ }^{7}$ Increased infection rates have also been described after discharge from hospitals, ${ }^{10}$ suggesting that screening programs have long term benefits as well. The lack of data on infections due to MRSA during the study period is a drawback in this study. Further research into identifying risk factors associated with MRSA colonization on admission would help in establishing a more focused screening programme than universal screening. 
The high rates of MRSA colonization at admission, together with the possibility of acquiring MRSA during their hospital stay led to increased infection control activity approximately 6 months after the onset of the study. The orthopaedic unit has a very high patient throughput with outpatient clinics being held in the same location. There are no facilities for patient isolation and cohorting of MRSA positive patients is not possible due to lack of sufficient beds, sharing of toilet facilities and a common treatment room. Education on hand hygiene was intensified. Imipenem which was used as surgical prophylaxis for prosthetic surgery was replaced with Teicoplanin. Antibiotic use was monitored and where possible restricted. There was no evidence that these activities had any effect on in-ward acquisition of MRSA during the period of the study.

\section{Conclusion}

The relatively high prevalence of MRSA carriage on hospital admission and acquisition during the hospital stay is of concern in health care settings where a heavy patient load and limited facilities pose challenges to establish screening, isolation, and other recommended infection control measures.

Conflict of interest. : None declared

Acknowledgement: We thank Champa Ratnatunge for assistance with statistics

\section{References}

1. De Leo FR, Otto M, Kreiswirth BN, Chambers HF. Community-associated meticillinresistant Staphylococcus aureus. The Lancet 2010; 75(9725):1557-1568. http://dx.doi.org/10.1016/S0140-6736(09)61999-1

2. Chambers HF. The Changing Epidemiology of Staphylococcus aureus? Emerging Infectious Diseases 2001; 7(2):178-182. http://dx.doi.org/10.3201/eid0702.010204

3. Safdar N, Bradley EA. The risk of infection after nasal colonization with Staphylococcus aureus. Am J Med. 2008 ; 121(4):310-315. http://dx.doi.org/10.3201/eid0702.010204

4. Yano K, Minoda Y, Sakawa A, Kuwano Y, Kondo K, Fukushima W, Tada K. Positive nasal culture of methicillin resistant Staphylococcus aureus (MRSA) is a risk factor for surgical site infection in orthopaedics. Acta Orthopaedica 2009; 80(4):486-490. http://dx.doi.org/10.3109/17453670903110675

5. Hassan K, Koh C, Krunaratne D, Hughes C, Giles SN. Financial implications of plans to combat methicillin resistant Staphylococcus aureus (MRSA) in an orthopaedic department. Ann R Coll Surg Engl 2007; 89:668-671. doi 10.1308/003588407X209400

6. Corea E, de Silva T, Perera J. Methicillin Resistant Staphylococcus aureus: prevalence, incidence and risk factors associated with colonization in Sri Lanka. Journal of Hospital Infection 2003; 55:145-148. http://dx.doi.org/10.1016/S0195-6701(03)00256-1

7. Davis KA, Stewart JJ, Crouch HK, Florez CE, Hospenthal DR. Methicillin-Resistant Staphylococcus aureus (MRSA) nares colonization at hospital admission and Its effect on subsequent MRSA infection. Clinical Infectious Diseases 2004; 39:776-782.

doi: $10.1086 / 422997$ 
8. Santos HB ,Machado DP, Camey SA, Kuchenbecker RS, Barth AL, Wagner MB. Prevalence and acquisition of MRSA amongst patients admitted to a tertiary-care hospital in Brazil. BMC Infectious Diseases 2010; 10:328 http://dx.doi.org/10.1186/1471-2334-10328

9. Price CS, Williams A, Philips G. Staphylococcus aureus Nasal colonization in preoperative orthopaedic outpatients. Clin Orthop Relat Res 2008; 466:2842-2847. http://dx.doi.org/10.1007/s1 1999-008-0337-x

10. Huang SS, Platt R . Risk of Methicillin-Resistant Staphylococcus aureus Infection after previous infection or colonization. Clinical Infectious Diseases 2003; 36:281-5 http://dx.doi.org/10.1086/345955 